\title{
New Materials Design Using Excitonic Quantum Effects: Applications for Fuel Cells, Catalysts, Superconductive and Bioactive Materials
}

\author{
Prof. Vladimir B. Kopylov, Paul R. Leonard* \\ Cascade Catalysts, Inc., $1090-14^{\text {th }}$ Street, West Vancouver, BC, V7T 2R6 \\ *E-mail correspondence: paul.r.leonard@gmail.com
}

\begin{abstract}
This manauscript begins with a theoretical substantiation of the possibility of thermal (dark) generation of electronically excited states (excitons) in the structure of oxides as a fundamental quantum-chemical property that ensures their continuous activity. For the first time, experimentally, a macro-scale process flow and its quantum nature are proven using a wide range of tools. The mechanism, features of the chemical structure of electron-hole components, and their role in the formation of catalytic properties have been extensively studied. The application of these new principles for the creation of highly efficient catalysts, energy generation and energy storage devices has been evaluated.
\end{abstract}

\section{Introduction}

A specific feature of oxides and materials based upon them is that the reactivity, transport, magnetic and mechanical properties are not uniquely determined by their chemical and phase composition. This is largely due to the presence and synergism of local excitations. Unfortunately, determination of the real structure of excited transition states has not been the focus despite new possibilities in modern physicochemical experimentation and progress in theoretical quantum-chemical developments. In solidstate chemistry, it corresponds to the problem of structural and chemical criteria for directed and reproducible synthesis. The answer to this question can be obtained by solving the complex problem of revealing the mechanism of thermal (dark) generation of electronically excited states, the features of their chemical structure and stability conditions. The effect of spatio-temporal coherence of the elementary stages of chemical reactions, which is most clearly manifested in large-scale catalytic action, anomalous 
mass and electrical transfer-- as well as the special properties of low-dimensional heterostructures, indicate the fundamental role of excitons. Modern data show [1] that active reaction states upon thermal and photoexcitation have many features in common; the donor-acceptor properties of molecules control reactions of both types. At the same time, theoretically and experimentally, the idea of the fundamental importance of thermal electronic excitation in the acts of breaking and forming bonds is being developed as a factor that ensures overcoming the symmetry ban corresponding to the WoodwardHoffman rule [2].

Oxide systems include the largest number of compounds that have high and highly different chemical activity with a relatively small difference in phase and chemical composition. At the same time, the problem of disclosing the mechanism of the formation of a high universal catalytic activity of dilute complex oxide systems based on oxides of $\mathrm{p}-, \mathrm{d}-$, f-elements and various ceramic supports has been and remains relevant. Its most important and fundamental aspects are the determination of the chemical structure of the components of excited electron-hole states in the cationic and anionic sublattices, analysis of their cooperative properties, as well as the role of the surface, interphase boundaries, and the size of structural units in the processes of formation, association, and relaxation of excitations.

Until now, there is practically no systematic information on the chemical features of the structure of electronically excited states and their effect on the physicochemical properties of oxides.

The purpose of this work was to determine the effect, scale, and mechanism of thermal generation of electronically excited states, their chemical structure and role in the formation of the physicochemical properties of oxides.

This goal was achieved by solving the following tasks:

1. Development of a theoretical concept of thermal generation of electronically excited states and their fundamental role in the formation of physicochemical properties of oxides.

2. Development of experimental methods for the analysis of excited states in condensed and gaseous media in a wide temperature range. 
3. Determination of the mechanism of the formation of dark excitations, the role in this process of the chemical and supramolecular structure of oxides (surface, interphase boundaries, dislocations, point defects).

4. Determination of the role of excited states in the formation of the catalytic activity of the supported catalytic systems for the processes of deep oxidation of hydrocarbons.

5. Assessment of the effect of exciton states on the chemical activity of oxides in reactions of surface modification and heterogeneous synthesis.

To solve the set tasks, the following were developed:

- Gas chromatographic and microcalorimetric techniques for the quantitative analysis of singlet forms of molecular oxygen emitted by oxides.

- Spectroscopic technique of analysis in a wide temperature range of the chemical structure of electronically excited and metastable states of oxides.

- New methods for the analysis of adsorption properties, parameters of the porous structure and density of solids.

- Photoacoustic method for analyzing the parameters of relaxation of vibrational excitations in the processes of laser destruction of materials.

- A new method for measuring magnetic susceptibility.

Based on the results obtained, new methods were developed for obtaining complex oxide catalytic systems for the processes: partial oxidation of carbon tetrachloride [3], deep oxidation of hydrocarbons [4], and new methods for the synthesis of film condensed carbon structures on the surface of oxides [5].

\section{Structural and chemical factors of thermal activation of electronic structure in molecular systems}

A quantum-chemical assessment of the electronic structure of oxygen polyhedron elements shows that the wave functions of excited states (configuration interaction) always enter the correct wave function of the ground state to varying degrees [1]. A characteristic feature of the oxygen complex element is a significant amount of binding $\pi$-type MOs. An increase in the order of elemental oxide bonds creates barriers to the rotational relaxation of vibrational, thermally initiated excitations, and thus provides one 
of the main conditions for energy accumulation. A relatively large fraction of $\pi$-orbitals corresponds to polyhedra with tetrahedral symmetry.

Singling out the role of local bond stresses and cooperative deformations of structural elements as a source of energy for the process of thermal activation of the electronic structure, one can imagine a formal sequence of its stages: structural deformation $\rightarrow$ vibrational excitation $\rightarrow$ vibronic interactions $\rightarrow$ electronic excitation and its structural and chemical consequences.

An assessment of the scale of interaction of electronic and vibrational degrees of freedom in molecular systems made it possible to conclude that, in the absence of an external source of excitation, the internal resources of a condensed system, due to the interaction of elementary oscillators, can provide the generation of excited states [6]. Quantum-mechanical analysis revealed isolation in the nature of the behavior of electronic excited oscillators [7], which served as a key to their spectral identification.

\section{Redox reaction - a source of energy for the formation of electronic excitations in oxides}

The thermal model of the formation of electronic excitations within the framework of the band theory raises the question of the internal energy source of this process. From a chemical point of view, we are talking about a redox reaction, during which a Mott exciton appears - two atoms with opposite excess charges. The excitation energy corresponds to the transformation potential [8]:

$$
\mathrm{W}=\mathrm{W}^{-}+\mathrm{W}^{+}+\Delta \mathrm{W},
$$

where $\mathrm{W}^{-}$is the energy spent on the introduction of an electron into the lattice; $\mathrm{W}^{+}$is the energy spent to remove an electron; $\Delta \mathrm{W}$ is the interaction energy between an electron and a hole, for free charge carriers $\Delta \mathrm{W}=0$. In the $\mathrm{M}^{+} \mathrm{O}^{-}$oxide lattice, the state $\mathrm{M}$ corresponds to the electron, and the state $\mathrm{O}$ corresponds to the hole. The empirical formula of Hilsch and Pole agrees better with experiment than others:

$\mathrm{W}=\left(\mathrm{e}^{2} / \mathrm{a}\right) \mu+\mathrm{E}-\mathrm{I}$, where $\mathrm{e}$ is the electron charge; $\mathrm{a}$ is the shortest distance between ions; $\mu$ is the Madelung coefficient; I is the ionization energy of the M atom; E - energy of affinity for an electron of an oxygen atom. 
Application of this formula to estimate the dependence of the energy of WannierMott excitons on their size (na) showed that in the case of $\mathrm{KCl}$ the exciton energy tends to zero and acquires small negative values only starting from $n=17$. In copper oxide, this result is achieved already at $\mathrm{n}=2$ and the magnitude of the exoenergetic effect is large and quite quickly reaches values close to the limiting value $\left(\mathrm{W}_{\mathrm{n} \rightarrow \infty}=-6.25 \mathrm{eV}\right)$.

The results obtained allowed us to conclude that the redox process of the formation of excitons with different radii $(n \geq 2)$ includes exoenergetic stages. Due to the latter, a highly efficient transformation of distortions of structural polyhedra into electronic excitations of the exciton type can be realized.

The analysis of the spectrum of one-electron excitations plays a special role in the construction of the theory of high-temperature superconductivity, which led to the development of a large number of exciton models [9], including the model of collective electronic excitations associated with charge transfer $\mathrm{Cu}^{2+} \mathrm{O}^{2-} \rightarrow \mathrm{Cu}^{1+} \mathrm{O}^{1-}$. A comparative analysis of $\mathrm{T}_{\mathrm{c}}$ and vibrational spectra of a number of oxide compounds $\left(\mathrm{La}_{2} \mathrm{CuO}_{4}, \mathrm{YBa}_{2} \mathrm{Cu}_{3} \mathrm{O}_{7}, \mathrm{BaBiO}_{3}, \mathrm{HgCaBaCuO}\right)$ showed [10] that in the low-frequency region for all samples, intense bands are recorded at 254, 280, 302, and $502 \mathrm{~cm}^{-1}$. The peak at $302 \mathrm{~cm}^{-1}$ is emission. The high intensity of the lines and the Zeeman splitting make it possible to classify them as acts of dissociation and bonding of electron-hole pairs. In this case, the optical density of the peaks at $502,302 \mathrm{~cm}^{-1}$ and $\mathrm{T}_{\mathrm{c}}$ change symbatically, and at 254 and $280 \mathrm{~cm}^{-1}$ - antibatic.

A characteristic feature of the spectra of high-temperature superconductors is a sharp drop in absorption (luminescence) during the transition to the superconducting state for frequencies $v<500 \mathrm{~cm}^{-1}$. In this case, as the temperature decreases, the intensity of the luminescence band increases with a maximum of about $300 \mathrm{~cm}^{-1}$, which corresponds to the formation of a superconducting gap, and, which has not yet had a satisfactory explanation, is recorded for some samples at temperatures above critical.

Registration of a well-resolved, intense spectrum of bands of the ground and electronically excited states of neutral and charged oxygen dimers $\left(\mathrm{O}_{2}, \mathrm{O}_{2}{ }^{2-}\right)$ reveals the nature of the previously discovered phenomenon of anomalously high frequencies and line intensities in the Raman spectra of high-temperature oxide superconductors [11]. Thus, the model of collective electronic excitations finds its detailed confirmation. 
The initiation of the process of one-electron transfer by overcoming the energy barrier at the level of the electron affinity energy of oxygen atoms $(\sim 1.47 \mathrm{eV})$ is presented as a result of vibronic amplification of vibrational disturbances within the framework of the cooperative Jahn-Teller effect and the multiphoton mechanism of energy accumulation [7]. The latter is due to the efficiency of the V-R-T relaxation process.

Comparison of the level of values of the photoacoustic relaxation parameter $\Omega$ for oxides and high-polymer organic compounds, which have a relatively wide set of $\mathrm{V}$ $\mathrm{R}$ - $\mathrm{T}$ degrees of freedom (polyethylene, polypropylene), shows that the specific acoustic response of the latter is almost an order of magnitude higher than oxides [12]. The use of high-modulus organic polymer materials (Kevlar, SVM) as objects of study, whose anomalous physicomechanical properties are associated with the formation of effective $\pi$-conjugation in the polymer chain due to keto-enol rearrangements of the quinoid type, demonstrates a significant drop in the value of the relaxation efficiency parameter $\Omega$ to values characteristic of the structure of oxides. The nature and rate of formation of monomeric volatile products of laser destruction of polymeric materials made it possible to quantitatively estimate the efficiency of the transformation of vibrational excitation into electronically excited states of chemical bonds. According to this criterion, polymers are divided into three groups. The first includes heat-resistant - aramid and arimide fibers, which are characterized by high values of the rate constants of destruction and the formation of condensed carbon structures. The second group includes oxygen-containing polymers: aliphatic amides, materials of the cellulose group, polyethylene terephthalate. The release rate of volatile products is noticeably lower. The third group includes materials based on linear polymers such as polyacrylonitrile and polyethylene. The effect of laser radiation on these polymers is practically not accompanied by the formation of volatile degradation products; it has the character of melting and evaporation. Thus, the role of the rotational degrees of freedom in the relaxation of selectively initiated vibrational excitations is manifested and confirmed. 


\section{Chemical structure of cationic and oxygen defects sublattices of oxides}

Analysis of the literature data showed that the most complete and detailed study of the properties of oxides in this state (high synthesis temperature, low level of impurity concentration), for which the influence of impurity atoms is negligible and dominant are lattice defects that appear as a result of intrinsic, thermodynamically favorable, disordering. It can be stated with confidence that the most studied are such features of the chemical behavior and such properties of oxides, which are due to their nonstoichiometry, and of the wide variety of defects present in oxides, those dominant in nonstoichiometric phases.

In the framework of this study, it was of particular interest to consider the process of association of defects, which forms a particular type of distribution of metal atoms in the oxide structure. In particular, such arrangement of atoms is of particular interest when they are linked directly by MM bonds or through an oxygen bridge into so-called clusters characterized by a set of extraordinary properties that attract attention from a practical and theoretical point of view.

The interaction between defects (attraction, repulsion) observed above the threshold concentration values $\left(\sim 10^{17} \mathrm{~cm}^{-3}\right)$ leads to rearrangement of neighboring atoms and groups and to the formation of superstructures - long-range order structures [13]. In most oxides, the formation of defects and superstructure easily occurs in the presence of impurities, but is difficult in pure oxides. This is especially true for oxides with highly symmetric coordination (most often octahedron) and close packing of oxygen ions, such as oxides of $\mathrm{Co}, \mathrm{Ni}, \mathrm{Fe}$. It is believed that almost no superstructures are formed here. Another group consists of oxides Mo, W, V, Nb, Ta with an irregular structure, asymmetric arrangement of oxygen ions around metal ions, looseness in the packing of metal - oxygen polyhedra, forming a large number of oxides of complex stoichiometric composition, each of which is not a separate thermodynamically stable phase (blocks with ordered structure).

Disordering models (according to Andersen) [14] occupy an intermediate position between the two limiting hypothetical cases. The first of them corresponds to the highest degree of short-range and long-range order of defects, which leads to the replacement of 
the nonstoichiometric phase by a group of ordered structures close in composition with an insignificant concentration of defects. The second corresponds to a statistically random distribution of point defects. Between these extreme cases, there are states corresponding to the formation of associates and clusters, as well as to the partial disordering of superstructures. A special place for understanding the nature of compounds of variable composition, in general, and nonstoichiometric oxides of transition metals, in particular, is occupied by the thermochemical and magnetochemical studies of Aria [15], which to some extent anticipated the ideas of supramolecular chemistry about the decisive significance for the nature of solids not of structural defects, but of character interatomic bonds. It was he who came up with the idea of the microheterogeneous structure of oxides, put forward long before direct structural evidence of the formation of clusters in wustite and other nonstoichiometric oxides was obtained. Analysis of the magnetic susceptibility of solid solutions $\mathrm{FeO}-\mathrm{MgO}, \mathrm{CoO}$ $\mathrm{MgO}, \mathrm{NiO}-\mathrm{MgO}$ confirmed the presence of segregated metal atoms due to the formation of strong M-M bonds.

The presence in oxides of a number of dimeric and oligomeric oxygen compounds $\mathrm{O}_{2 \text { ads. }}, \mathrm{O}_{2}^{-}, \mathrm{O}_{2}{ }^{+}, \mathrm{O}_{3}{ }^{2-}, \mathrm{O}_{3}, \mathrm{O}_{3}^{-}, \mathrm{O}^{4-}$ is mainly associated with the occurrence of sorption processes. The possibility of the formation of a peroxide form with a covalent bond - $\mathrm{O}$ $\mathrm{O}-\mathrm{M}$ is relatively rarely assumed. Such compounds were noted for molybdenum oxides and HTSC complex oxide ceramics [16].

Earlier it was observed that the emission of singlet oxygen from the surface of solids can also occur in dark conditions. This ability was possessed by partially reduced $\mathrm{V}_{2} \mathrm{O}_{5}, \mathrm{MO}_{3}$, thin films of nonstoichiometric spinel $\mathrm{Co}_{3-\mathrm{x}} \mathrm{O}_{4}$, and complex oxide catalytic systems (oxides Li, Sn, P) [17].

\section{Analysis of thermal emission activity of oxides}

\section{thermal desorption spectra and specific activity anomalies}

Gas chromatographic analysis of the thermal desorption activity of silicate glasses (pyrex, quartz) [18], silica gels, aluminum oxides, aluminosilicates of cordierite structure, as well as platinum catalysts based on them showed [19] the extreme nature of the change in thermal emission with increasing temperature. This was most clearly manifested in the 
behavior of glasses. The thermionic emission spectra exhibit the presence of two sharp maxima with a characteristic identical temperature range $\left(\Delta \mathrm{T}=200^{\circ} \mathrm{C}\right)$, which indicates a certain structural analogy of the centers that determine thermionic emission.

Presumably, strained oxygen "bridges" combining surface silicon and boron - oxygen (in the case of Pyrex) tetrahedra (the bond of two boron - oxygen tetrahedra is unstable in accordance with the Levenshtein rule) can be considered as such. The stress caused by the "emergence" of tetrahedra to the surface is understood not only the possibility of strong distortion of the $\mathrm{Si}-\mathrm{O}$ - $\mathrm{Si}$ angles, B - O - Si and bond lengths Si - O, B - O, as well as an increase in the effective charge of bridging oxygen; in this case, the stability of oxygen in strained siloxane bonds is much higher than that in the B - O - Si bridges, which determines the sharp difference in the temperatures of their destruction. The first, in order of increasing temperature maximum, can be attributed to the homolytic elimination of the most unstable oxygen, which is common to two distant tetrahedra connected by vertices:

$$
\equiv \mathrm{B}^{-}-\mathrm{O}^{*}-\mathrm{Si} \equiv \leftrightarrow \equiv \mathrm{B}+\cdot \cdot \mathrm{Si} \equiv+\mathrm{O}
$$

the second - to a similar process for the case of a stressed bond of two surface tetrahedra having a common edge:

$$
=\mathrm{B}^{-}=\mathrm{O}_{2}=\mathrm{Si}=\leftrightarrow \equiv \mathrm{B}-\mathrm{O}-\cdot \cdot \mathrm{Si} \equiv+\mathrm{O}
$$

The proposed variant of "assignment" of thermionic emission maxima implies greater stability of B - O - Si bonds in the case of (boron) silicon-oxygen tetrahedra connected by a common edge; it is assumed that in this case the proximity of the polyhedra provides less "stretched" and, therefore, less stressed bonds.

Equilibria similar to those indicated in the diagrams are also realized in the case of strained siloxane bonds on the surface of quartz glass at high temperatures; The same interval of $200^{\circ} \mathrm{C}$ separating the two maxima in the thermal emission spectra corresponds to the difference in the activation energies of the processes reflected by schemes (1) and (2), which is close for Pyrex and silica glass.

Estimation of the concentration of strained surface fragments according to the data for quartz glass gives a value of $1.5 \cdot 10^{14}-1.8 \cdot 10^{14} \mathrm{~cm}^{-2}$, which is in good agreement with the known one $\left(\sim 10^{14} \mathrm{~cm}^{-2}\right)$. 
The maxima in the thermionic emission spectra are observed at a relatively high average background level of $0.10-0.15 \mathrm{nmol} / \mathrm{cm}^{2}$, and in the case of silica glass in the region of $100-400^{\circ} \mathrm{C}$, a broad blurred band stands out. Its presence is evidently due to the fact that the real surface of glasses is characterized by a wide variety of centers with a close to continuous distribution of oxygen binding energy.

The values of the activation energies $E_{a}{ }_{\max }(38.3 ; 25.1 \mathrm{~kJ} /$ mol - quartz and $13.4 ; 23.5 \mathrm{~kJ} / \mathrm{mol}$ - Pyrex) indicate that the rate of thermal emission is limited by diffusion. Hence it follows that the formation of molecular oxygen from atoms does not occur in the gas phase, but as a result of their structural recombination.

In the course of the experiments, the effect of the influence of the mass of the samples on the magnitude of the thermionic activity and the form of the spectra was established, which was most clearly manifested in the samples with a relatively small specific surface area of cordierite $\left(\mathrm{MgAl}_{4} \mathrm{Si}_{5} \mathrm{O}_{18}\right)$ and $\alpha-\mathrm{Al}_{2} \mathrm{O}_{3}$. The analysis of the obtained dependence was based on the assumption that $\mathrm{O}_{2} *$ molecules in excited singlet states are formed as a result of sequentially proceeding processes of thermally activated emission and recombination of oxygen atoms. For the process diagram:

$2[\mathrm{AlOSi}] \leftrightarrow 2$ [AlSi] $+\mathrm{O}_{2}$, the initial concentration of strained fragments of the structure was taken as $[\mathrm{AlOSi}]=1$; then the current concentration: $[\mathrm{AISi}]=\mathrm{X} ;[\mathrm{AlOSi}]=(1-\mathrm{X})$; $\left[\mathrm{O}_{2}\right]=0.5 \mathrm{X}$. The equation of equilibrium emission - sorption had the expression:

$$
\mathrm{K}_{\mathrm{e}} \cdot(1-\mathrm{X}) 2=\mathrm{k}_{\mathrm{s}} \cdot 0.5 \cdot \mathrm{X}^{3},
$$

where $\mathrm{k}_{\mathrm{e}}$ and $\mathrm{k}_{\mathrm{s}}$ are the rate constants of the emission and sorption, respectively. ks corresponds to the rate constant of heterogeneous deactivation of excited molecules:

$$
\mathrm{k}_{\mathrm{s}}=\varepsilon \cdot \mathrm{S} \cdot \mathrm{v} / \mathrm{V} \cdot 4=\mathrm{m} \cdot \mathrm{k}_{\mathrm{s}}{ }^{\circ},
$$

where $\mathrm{S}$ and $\mathrm{V}$ are the surface and volume of the reaction system, respectively, $\mathrm{v}$ is the average thermal velocity of excited molecules, $\varepsilon$ is the accommodation coefficient (the coefficient of death or deactivation) of singlet oxygen.

Since $\mathrm{S}=\mathrm{m} \mathrm{S}_{\mathrm{sp}}$ at $\mathrm{k}_{\mathrm{s}}{ }^{\circ} / \mathrm{k}_{\mathrm{e}}=\mathrm{A}$, the original equation is transformed into the form:

$$
\mathrm{X}^{3}-2 \mathrm{X}^{2} / \mathrm{mA}+4 \mathrm{X} / \mathrm{mA}-2 / \mathrm{mA}=0 \text {. }
$$

The solution of the equation made it possible to establish a functional relationship between the specific oxygen emission and the oxide mass:

$$
\mathrm{X}=\left[5.4 \cdot(\mathrm{A} \cdot \mathrm{m})^{2}+0.8 \cdot \mathrm{A} \cdot \mathrm{m}-1.1\right] / \mathrm{A} \cdot \mathrm{m}(12 \mathrm{~A} \cdot \mathrm{m}-4) .
$$


Sample plots of the function for a number of fixed values of the parameter A correlated satisfactorily with the experimental results.

Mild hydrothermal treatment, which homogenizes the surface, removing local stresses, significantly reduces the thermionic activity and the scale of the effect of the influence of the sample mass.

A linear drop in the specific (per surface unit) thermionic activity with an increase in the specific surface area of the samples indicated the bulk origin of labile oxygen forms.

At the same time, a sharp increase in the activity during surface modification (deposited catalytic contacts) indicates that the surface state is one of the limiting factors of the process.

\section{Chromatographic separation and spectral analysis of desorbed oxygen}

In the formulation of the problem of gas chromatographic separation of the basic and excited forms of oxygen [20], a significant difference in their sorption properties was taken into account. The hydrothermal treated $\mathrm{NaX}$ zeolite was used as a sorbent. Chromatograms were recorded in the samples for the presence of three and Bode components.

To calculate the chromatographic retention time of triplet molecules, the Frenkel equation was used in the form: $\tau_{\text {out }}=\tau_{0 \text { out }} \cdot \exp \mathrm{Q} / \mathrm{RT}$, where $\tau 0$ out is the minimum delay time of a non-adsorbed gas, corresponding to the time it passes through the free volume of the column, $\mathrm{Q}$ is the heat of adsorption. Taking into account the fact that the temperature of the excited molecules does not coincide with the temperature of the thermostat, but is determined by the energy of their excitation $\left(\mathrm{E}^{*}\right)$, the following equation was used to calculate the retention time of singlet molecules: $\tau^{*}{ }_{\text {out }}=\tau_{0 \text { out }} \cdot \exp \mathrm{Em} / \mathrm{E} *$, where $\mathrm{Em}$ is the activation energy of surface migration, which limits the deactivation of molecules. The calculation results showed good agreement with the experimentally determined retention times.

IR spectrometric analysis of gas samples by the radiative and absorption activity of vibrational transitions of triplet $\left(1557-1460 \mathrm{~cm}^{-1}\right)$ and singlet molecules 
$\left(1457-1290 \mathrm{~cm}^{-1}\right)$ of oxygen confirmed the calculated identification of the peaks.

The results of the analysis showed that the surface of borosilicate glass in the entire temperature range is the source of the first form of singlet oxygen $\left({ }^{1} \Delta_{\mathrm{g}}\right)$, quartz glass is the source of the highly excited state ${ }^{1} \Sigma_{\mathrm{g}}+$ (molar ratio of the main and excited forms of emitted oxygen $\mathrm{M} \approx 1$ ). Analysis of gas samples thermally desorbed from the surface of the alumina-platinum catalyst showed an anomalously high emission value and the simultaneous presence of three forms of molecular oxygen. The singlet form ${ }^{1} \Delta_{\mathrm{g}}$ was predominant.

\section{Adsorption-calorimetric method for the analysis of thermionic activity of deposited cobalt and molybdenum oxides}

The difference of more than an order of magnitude in the heats of adsorption of singlet and triplet oxygen molecules on the surface of activated carbon determined the possibility of quantitative determination of each of the forms according to the data of the calorimetric experiment [21]. The experimental setup developed on the basis of a heatconducting microcalorimeter using anisotropic thermoelements from a bismuth single crystal provided a sensitivity of $10^{-7} \mathrm{~mol}$. The analysis of oxygen thermal emission during $\mathrm{HgO}$ thermolysis showed that singlet oxygen molecules ( $90 \%)$ predominate in thermally emitted samples, the process has a two-peak character, which corresponds to the scheme:

$$
\begin{aligned}
& 4 \mathrm{HgO} \leftrightarrow 2 \mathrm{Hg}_{2} \mathrm{O}+\mathrm{O}_{2} * \\
& 2 \mathrm{Hg}_{2} \mathrm{O} \leftrightarrow 4 \mathrm{Hg}+\mathrm{O}_{2} * .
\end{aligned}
$$

Comparison of the results obtained in the study of the formation of the structure of deposited (by impregnation) cobalt and molybdenum oxides on the surface of silica gels and aluminosilica gels using diffuse reflection electron spectroscopy, density measurements, and the adsorption-calorimetric method made it possible to establish that the singlet oxygen emission maxima correspond to the formation of metal clusters. The presence of an alumina modifying layer significantly increases the intensity of the process and expands the temperature limits of extreme activity. The modifying layer promotes the clustering process and provides an increase in oxygen mobility. 


\section{Thermodynamic assessment of the nature of the thermionic emission process}

The nonlinear cubic character of the functional relationship between the specific amount of desorbed gas and the mass of the samples made it possible to draw an analogy between the considered heterogeneous system and the vapor - liquid equilibrium, i.e., van der Waals isotherms. A consequence of this analogy was the hypothesis of the critical nature of the process under consideration, which may be correct if quasi-liquid phases are formed in the oxygen sublattice.

From the basic equation of thermodynamics of irreversible processes for anisotropic heterogeneous systems [22]:

$$
\sigma=\mathrm{J}_{\mathrm{ox}} \operatorname{grad}\left(-\mu_{\mathrm{ox}} / \mathrm{T}\right)+\Sigma_{\mathrm{i}}\left(\mathrm{A}_{\mathrm{i}} / \mathrm{T}\right) \mathrm{V}_{\mathrm{i}},
$$

where $\mathrm{J}_{\mathrm{ox}}$ is the diffusion flow of oxygen; $\mu_{\mathrm{ox}}$ is the chemical potential of oxygen; $\mathrm{Ai}$ - affinity of chemical reaction $\mathrm{i} ; \mathrm{V}_{\mathrm{i}}$ is the rate of chemical reaction $\mathrm{i}$; as well as oxygen thermionic emission schemes:

$$
\begin{aligned}
& \mathrm{Si}_{\mathrm{m}} \mathrm{O} 2_{\mathrm{m}} \leftrightarrow \mathrm{SimO}_{2(\mathrm{~m}-\mathrm{n})}+\mathrm{nO}_{2} \\
& \mathrm{Si}_{\mathrm{m}} \mathrm{O} 2_{\mathrm{m}} \leftrightarrow \mathrm{SimO}_{2(\mathrm{~m}-\mathrm{n})}+\mathrm{nO}+\mathrm{n} / 2 \mathrm{O}_{2},
\end{aligned}
$$

a functional relationship is established between the concentration of molecular oxygen and oxygen atoms, which are included in the system of point structural defects:

$$
\mathrm{C}_{\mathrm{O} 2}=\mathrm{C}_{\mathrm{O}}^{2(\mathrm{~B} / \mathrm{n}-1)} \cdot \mathrm{f}^{-0.5 \mathrm{n}}
$$

where $\mathrm{B}=\mathrm{D}_{02} \mathrm{~d} \rho / \mathrm{dx} \cdot \Delta \mathrm{x} \cdot \mathrm{V}$; $\mathrm{f}$ is a function of temperature, $\mathrm{n}$ is an integer.

The application of the Shklovsky-de Gennes model [23] within the framework of the percolation theory made it possible to establish the dependence of the concentration of emitted molecular oxygen on the sample mass:

$$
\mathrm{C}_{\mathrm{O} 2}=\left[\mathrm{C}_{\mathrm{O} c}+0.54 \cdot(\mathrm{k} \cdot \mathrm{m})^{-0.3}\right]^{2(\mathrm{~B} / \mathrm{n}-1)} \cdot \mathrm{f}^{-0.5}
$$

where $\mathrm{C}_{0_{\mathrm{c}}}$ is the percolation threshold; $\mathrm{k}=\mathrm{N}_{\mathrm{a}} / \mathrm{M}$. Thus, this confirms the hypothesis that the presence of structural excitations and the emission of singlet oxygen molecules are characterized by equilibrium:

quasi-liquid condensate (associates) of excited states - gas. 


\section{Manifestation of electronically excited states in the chemical structure and activity of oxides}

Data on the analysis of the components of exciton states in the structure of oxides, in general, and silicon oxides in particular, are practically absent in the modern literature. This is due to experimental difficulties in obtaining high-resolution vibrational spectra of solid samples and in assigning low-intensity spectral bands that are recorded against the background of fundamental absorption. Nevertheless, the use of modern high-resolution instruments of traditional IR, Fourier and Raman spectroscopy made it possible to obtain information on the nature and structural-chemical characteristics of stationary excitations.

\section{High-frequency anomaly in the spectra of silicon oxides}

The region of core vibrations of silicon oxides and their derivatives lies in the range $1200-700 \mathrm{~cm}^{-1}$. The center of gravity of the broad fundamental band ${ }_{v}$ as (si - o - si), which is characteristic of all polysiloxanes and silicas, is recorded at about $1100 \mathrm{~cm}^{-1}$. The short- and long-wavelength branches of the absorption curve contain characteristic series of well-resolved fine structure. In the range of frequencies close, but higher than the frequencies of maximum absorption of structural vibrations, at least ten narrow bands are observed at 1262, 1250, 1245, 1240, 1237, 1234, 1229, 1227, 1223, 1220, 1215, 1205 $\mathrm{cm}^{-1}$. In this case, the local absorption maximum of this series is at $1230-1234 \mathrm{~cm}^{-1}$.

The upper frequency limit of the manifestation of natural vibrations of the connection $\mathrm{Si}-\mathrm{O}$ is limited by the frequency of the main transition in the molecular, isolated state of the electronic term $\mathrm{X}^{1} \Sigma^{+}$at $v_{1-0}=1229 \mathrm{~cm}^{-1}$. Therefore, the series experimentally recorded for all samples (glass, single crystal, aerosil, silica gel) in the range $1229-1205 \mathrm{~cm}^{-1}$ was assigned to the vibrational transitions of the isolated $\mathrm{Si}$ - O state. The result obtained corresponds to the manifestation of the Berremen effect [24], an increase in the frequency of vibrations in microheterogeneous systems $\mathrm{SiO}_{2}\left(v_{1} \approx 1240 \mathrm{~cm}^{-1}\right)$, the nature of which is due to the presence of Coulomb excitons (excitations obeying Bose statistics) under conditions of a two-dimensional quantum well. The assignment of the band is confirmed by the registration of lines that correspond to the electronically excited states of the isolated $\mathrm{Si}$ - O oscillator:

${ }^{3} \Sigma^{+}(\mathrm{E} \sim 4 \mathrm{eV})-790 ;{ }^{3} \Pi_{0-2}(\mathrm{E} \sim 4 \mathrm{eV})-1000 ;{ }^{3} \Delta_{\mathrm{r}}(\mathrm{E} \sim 4.5 \mathrm{eV})-760 ;$ 
${ }^{1} \Delta(\mathrm{E} \sim 4.8 \mathrm{eV})-720 ;{ }^{1} \Pi(\mathrm{E} \sim 5 \mathrm{eV})-840 \mathrm{~cm}^{-1}$

\section{States of the anionic and cationic sublattices: role of the surface}

Analysis by vibrational and electronic spectroscopy of the chemical structure of defects in the oxygen sublattice of silicon oxides revealed the presence of a number of isolated dimeric and trimeric oxygen associates in the ground and electronically excited states: $\mathrm{O}_{2}, \mathrm{O}_{2}^{-}, \mathrm{O}_{2}{ }^{2-}, \mathrm{O}_{2}{ }^{+}, \mathrm{O}_{3}, \mathrm{O}_{3}^{-}$. The registration of high-intensity absorption bands in the electronic spectra of $\mathrm{SiO} 2$ at $\lambda_{\max }=250 \mathrm{~nm}$ (Hartley band) and $\lambda_{\max }=240 \mathrm{~nm}$ is in good agreement with the results of using vibrational spectroscopy [25].

The calculation of the concentration of trimeric associates of oxygen and superoxide groups using the known values of the molar absorption coefficients showed values at the level of $10^{17}-10^{19} \mathrm{~cm}^{-3}$.

The reduced states in the cationic state correspond to the oxidized states of the oxygen sublattice; the latter are manifested by vibrational transitions in the system of isolated $\mathrm{Si}$ - Si oscillators.

Comparison of the optical density of the characteristic lines in the IR spectra and the specific surface of the samples reveals the presence of a stable positive correlation of the latter with the concentration of silicon monoxide and isolated $\mathrm{Si}$ - $\mathrm{Si}$ bonds, as well as the ground and highly excited states of dimeric oxygen. For the first singlet state and ozone, the correlation is negative.

\section{Exciton states and cooperative effects}

Estimation of the energy of dissociation of excitons in silicon oxide by the formula:

$$
\mathrm{E}_{\mathrm{n}}{ }^{\mathrm{x}}=-\left(\mathrm{m}_{\mathrm{r}} / \mathrm{mn}^{2} \varepsilon^{2}\right) \mathrm{E}_{\mathrm{H}},
$$

where $\mathrm{E}_{\mathrm{H}}$ is the ionization energy of the hydrogen atom $(13.5 \mathrm{eV}), \varepsilon$ is the dielectric constant $(\varepsilon \mathrm{SiO}=4.6), \mathrm{m}_{\mathrm{r}}$ is the reduced exciton mass; showed that the maximum value $(\mathrm{n}=1)$ corresponds to a frequency of $\sim 500 \mathrm{~cm}^{-1}$.

A feature of the hydrogen-like levels of exciton states is the Zeeman splitting in a magnetic field [26]. The experiment showed that a series of intense narrow bands undergoing Zeeman splitting are recorded in the low-frequency spectral region for silica 
samples, with maxima at 510-500, 420, 350, 302, 290, 280, and $255 \mathrm{~cm}^{-1}$. The scale and clarity of recording the property under consideration made it possible to use the effect of splitting of bands in a magnetic field to identify similar states in the structure of oxides.

At concentrations ( $\mathrm{n}$ ) of exciton states in semiconductors, which correspond to the condition $\mathrm{na}^{3} \sim 1$ ( $\mathrm{a} \sim 10^{-6} \mathrm{~cm}$ is the effective radius of the Wannier-Mott exciton), their interaction becomes significant, in addition to the formation of an electron-hole liquid, free many-particle complexes, neutral exciton molecules (biexcitons) and excitonic ions (bound states of an exciton and a hole or an electron). The first, in silicon oxide, correspond to the oxidized states of oxygen in the form of molecular oxygen, ozone, the second - superoxide, peroxide groups, as well as positively charged oxygen associates. The concentration of these states, the order that was determined from thermal desorption and spectral data (estimated values of oxygen concentrations in semiconductor single crystals, corresponding to the onset of the association process $\sim 10^{17}-10^{18} \mathrm{~cm}^{-3}$ ), ensures that the above condition is met.

In the spectrum of A-300, in accordance with model concepts, high-intensity broadened emission bands with centers of gravity at $1050,1235,1370$, and $1750 \mathrm{~cm}^{-1}$ are clearly recorded. The position of the maxima of the emission bands corresponds to the heats of vaporization of dimers, tetramers $\left(\mathrm{O}_{4}^{+}, \mathrm{O}_{4}^{-}\right)$of oxygen and ozone. According to the results of computer simulation, the $\mathrm{O}_{4}{ }^{+}$ion cluster is characterized by an intense line at $1875 \mathrm{~cm}^{-1}$, and $\mathrm{O}_{4}^{-}-$at $1756 \mathrm{~cm}^{-1}$. Hence, it follows that the emission band and the characteristic absorption peak at $1750 \mathrm{~cm}^{-1}$ are most likely associated with the existence of the $\mathrm{O}_{4}{ }^{-}$ion cluster. The formation of an exciton condensate is confirmed by the registration of the size effect in the form of the dependence of the optical density of infrared luminescence on the particle size of silicon oxide. Among glasses and singlecrystal samples, single-crystal quartz stands out, the only one in this group exhibiting luminescent activity in the IR region, and at a level that corresponds to highly dispersed aerosils. This effect can be associated with the piezoelectric properties of a single crystal, which are manifested as a result of mechanical influences during the preparation of a sample for spectrophotometry and cause the appearance of superlattices in the structure. The latter significantly increase the concentration of excitons and the critical temperature of the exciton gas - condensate equilibrium. 
The cooperative, ordered character of the states, the condensation interaction of which causes infrared luminescence, is confirmed by the effect of its suppression by a constant magnetic field.

\section{The chemical structure of the excited states of silica at elevated temperatures}

An analysis of the spectra obtained in the temperature range of $298-1173 \mathrm{~K}$ for samples of Aerosil A-300 shows [27] that a sequential increase in temperature is accompanied by a gradual degradation and disappearance of emission bands observed at standard temperatures. In this case, the intensity of the luminescent band with the center at $3615 \mathrm{~cm}^{-1}$ increases and reaches its maximum value. Against its background, wellresolved absorption series appear, which correspond to the vibrational activity of hydroperoxide groups $-\mathrm{O}-\mathrm{O}-\mathrm{H}$. At the maximum experimental temperature of $1273 \mathrm{~K}$, the luminescence intensity drops sharply, while the absorption structure is retained. In accordance with the adopted model, the nature of the high-temperature luminescent bands corresponds to the process of condensation of polyexcitones, the degree of polymerization of which can be estimated from the chemical structure of oxygen associates.

As the temperature rises, an increase in the intensity of a series of absorption bands in the range $2060-2000 \mathrm{~cm}^{-1}$ is recorded in the spectrum, reaching a maximum value at $1273 \mathrm{~K}$. They correspond to highly excited $\mathrm{O}_{2}$ states and $\mathrm{O}_{3}$ overtone transitions.

Comparison of the change in the optical density of oxygen dimeric states with increasing temperature with the amount of desorbed oxygen made it possible to estimate the concentration of these compounds and the molar absorption coefficients. An increase in temperature up to $723 \mathrm{~K}$ is accompanied by a decrease in the optical density of the $\mathrm{D}_{1557}$ absorption band of stretching vibrations of triplet oxygen molecules. A further increase in temperature leads to an increase in the absorption intensity; at $1000 \mathrm{~K}$, the maximum optical density of the characteristic peak is observed. 
Using the formula

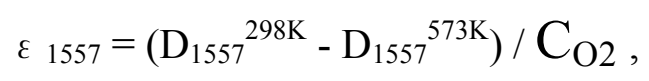

where $\mathrm{C}_{\mathrm{O} 2}$ is the specific amount of oxygen ( $\mathrm{mol} / \mathrm{g}$ ) desorbed in the range of 298$573 \mathrm{~K}$, at a constant density of the absorbing layer $\left(2 \mathrm{mg} / \mathrm{cm}^{2}\right)$ allows one to determine the value of the molar absorption coefficient $\varepsilon_{1557}=1.9610^{5} \mathrm{~g} / \mathrm{mol}=7.8410^{4}$ $\mathrm{cm}^{3} / \mathrm{mol}$. Calculation of the concentration at $298 \mathrm{~K}$ gives the value $\mathrm{C}_{\mathrm{O} 2}=3.3 \cdot 10^{-6} \mathrm{~mol} / \mathrm{cm}^{3} \approx 2 \cdot 10^{18} \mathrm{au} / \mathrm{cm}^{3}$. The minimum value at $573 \mathrm{~K}$ corresponds to a concentration of $=8.310^{-7} \mathrm{~mol} / \mathrm{cm}^{3} \approx 510^{17}$ at $\cdot$ unit $/ \mathrm{cm}^{3}$. The temperature dependence of the change in the optical density of the characteristic band of the main vibrational transition in singlet oxygen molecules $\left({ }^{1} \Delta_{\mathrm{g}}\right)$ has an extreme form with two maxima at 473 and $1173 \mathrm{~K}$. The molar absorption coefficient $\varepsilon_{1457}=2.6810^{5} \mathrm{~g} / \mathrm{mol}=1.0710^{5} \mathrm{~cm}^{3} / \mathrm{mol}$. The minimum concentration of singlet molecules is $\mathrm{C}_{0}{ }^{1 / 2}=5.610^{-7} \mathrm{~mol} / \mathrm{cm}^{-3}=3.410^{17} \mathrm{au} / \mathrm{cm}^{3}$, the maximum $-\mathrm{C}_{0}{ }^{1 / 2}=1.710^{-6} \mathrm{~mol} / \mathrm{cm}^{-3}=1.1 \cdot 10^{18} \mathrm{au} / \mathrm{cm}^{-3}$. Comparison of thermal desorption curves and dependences of oxygen concentration on temperature shows that the maxima of desorption correspond to minima of concentration and vice versa. In this case, the position of the concentration minima with respect to temperature coincides with the position of the optical density maxima of the emission bands. A distinctive feature of high-temperature spectra is the resolution and an increase in the intensity of the absorption band of highly excited states of dimeric oxygen $1 \Sigma_{\mathrm{u}}^{-}(\sim 4 \mathrm{eV})$ and $^{3} \Sigma_{\mathrm{u}}+(\sim 4.5 \mathrm{eV})$ in the range $769-775 \mathrm{~cm}^{-1}$. The temperature dependence of the optical absorption density has an extreme character with two maxima at 573 and $973 \mathrm{~K}$. The estimation of the concentration of states from the found value of the extinction coefficient gives $\mathrm{C}_{\Sigma \mathrm{u}}^{573}=3.1 \cdot 10^{-6} \mathrm{~mol} / \mathrm{cm}^{3}=1.9 \cdot 10^{18}$ at.u $/ \mathrm{cm}^{3}$ and $\mathrm{C}_{\Sigma \mathrm{u}}^{973}=1.8 \cdot 10^{-6} \mathrm{~mol} / \mathrm{cm}^{3}=1.1 \cdot 10^{18}$ at.u $/ \mathrm{cm}^{3}$. The high order of magnitude of the concentrations of highly excited states of small associates suggests the possibility of the formation of condensed phases such as excitonic liquids, the main structural element of which would be a biexciton. At $573 \mathrm{~K}$, the spectrum exhibits a high-intensity absorption series in the range $520-500 \mathrm{~cm}^{-1}$, which was previously assigned to the main transitions 
in the system of isolated $\mathrm{Si}$ - $\mathrm{Si}$ bonds, the envelope of which is an emission band. The position of the center of the band $\left(510 \mathrm{~cm}^{-1}\right)$ is close to the value of the photon frequency corresponding to the enthalpy of condensation of molecular oxygen $\left(544 \mathrm{~cm}^{-1}\right)$. In the low-frequency region, corresponding to isolated exciton levels, high-intensity luminescence bands are observed at 275 and $251 \mathrm{~cm}^{-1}$. A characteristic feature of the first band is the presence of an absorption peak at $271.5 \mathrm{~cm}^{-1}$ as a satellite, the frequency of which exactly corresponds to the ground transition in the electronically excited state $\left({ }^{3} \Sigma_{\mathrm{u}^{-}}, \mathrm{E}_{\mathrm{ex}}=3 \mathrm{eV}\right)$ of the isolated $\mathrm{Si}-\mathrm{Si}$ bond. Narrow intense exciton luminescence peaks are recorded at 419,332 and $266 \mathrm{~cm}^{-1}$. With an increase in the intensity of the band of stretching vibrations of hydroperoxide groups $\left(3610-3620 \mathrm{~cm}^{-1}\right)$, an increase in the absorption intensity of peaks in the range $700-600 \mathrm{~cm}^{-1}$, which correspond to vibrational transitions of peroxide groups, correlates.

The study of the magnetic susceptibility of silica samples in the temperature range of $298-1273 \mathrm{~K}$ showed that an increase in the specific surface area of the samples is accompanied by a significant increase in the diamagnetic response with maxima at 673 and $1173 \mathrm{~K}$. The stability of the temperatures at which the extrema of the diamagnetic susceptibility are recorded indicates the general nature of the observed effects. The latter correspond to the formation of an exciton condensate, which, in terms of its magnetic structure, is an orbital (non-spin) antiferromagnet consisting of itinerant (Bloch) electrons.

Comparison of the experimental curves of the temperature dependence of the average heat capacity of $\mathrm{SiO}_{2}$ with the Debye model revealed regions of excess $(\sim 10 \%)$, which correspond to the accumulation of electronically excited states.

\section{Features of catalytic carriers, catalysts and catalytic activity}

The most active modern high-performance catalytic systems for oxidation processes contain honeycomb block ceramics as carriers, which include aluminum oxides ( $\alpha-, \gamma-, \delta-, \theta-$ modifications) or aluminum-magnesium silicates of the cordierite structure type. Determination of the optical density of exciton lines and a comparative 
assessment of oxide carriers [28] shows that active alumina is released by an anomalously high concentration of exciton states with a minimum binding energy $\left(v=255,280 \mathrm{~cm}^{-1}\right)$, peroxide groups $\left(v=668 \mathrm{~cm}^{-1}\right)$, positively charged oxygen dimers $\mathrm{O}_{2}^{+}\left(\nu=1870 \mathrm{~cm}^{-1}\right)$, as well as the absence of superoxide groups $\mathrm{O}_{-2}\left(v=1140 / 1180 \mathrm{~cm}^{-1}\right)$. The last detail is significant, since superoxide groups, as intermediate ones, are a sign of a dead end in the processes of heterogeneous oxidation with the participation of oxygen. An equally significant difference is also the complete absence of characteristic emission bands (1040, 1240, 1380, $\left.1750 \mathrm{~cm}^{-1}\right)$, which are recorded in the spectra of other samples of the series under consideration, which is an indication of the small scale of condensation (stabilization) of exciton states.

Modification of the surface of cordierite and silica gel with a layer of aluminum oxide by impregnation with a solution of nitric acid salt in the first case is accompanied by a significant increase in the concentration of exciton states, in the second, no significant changes are observed. This is due to the similarity of the structures of the oxygen polyhedra elements of cordierite and $\gamma-\mathrm{Al}_{2} \mathrm{O}_{3}$, which contributes to the formation of the structure of defective spinel during salt thermolysis.

The linear correlation between the concentration (optical density) of superoxide groups and the optical density of the emission band at $1040 \mathrm{~cm}^{-1}$ indicates that the process of condensation with the indicated heat is due to the formation of monoexcitonic associates.

The linear correlation of the optical density of the singlet oxygen bands $\left(v^{1}{ }_{\Delta \mathrm{g}}=1456 \mathrm{~cm}^{-1}\right)$ and the $\mathrm{D}_{1250}, \mathrm{D}_{1380}, \mathrm{D}_{1750}$ emission lines correspond to condensation processes with the participation of biexcitons.

A characteristic feature of cordierite and active alumina, which is recorded with an increase in temperature, is anomalously intense absorption in the region of $3600 \mathrm{~cm}^{-1}$. The absorption character of the band is associated with the increased mobility of dimeric oxygen associates. The vibrational activity of hydroperoxide groups in this region is confirmed by an increase in the intensity of the absorption band of peroxide groups at $668 \mathrm{~cm}^{-1}$. 
An intense emission band is observed in the spectrum of silica gel at this frequency $\left(\sim 3600 \mathrm{~cm}^{-1}\right)$, which may indicate the formation of tightly bound oxygen associates $\left(\mathrm{O}_{4}{ }^{+}, \mathrm{E}_{\mathrm{CB}} \sim 35 \mathrm{~kJ} / \mathrm{mol}\right)$.

Determination of the temperature dependence of the magnetic susceptibility of active alumina and cordierite showed that in both cases an increase in the diamagnetic response is observed, which has maxima at 673 and $973 \mathrm{~K}$. The curve of the temperature dependence of the isobaric heat capacity of cordierite exhibits an anomaly at these temperatures, which may be a manifestation of phase transitions in the exciton condensate - gas system.

Comparison of the concentration of exciton states in the structure of transition element oxides by the optical density of characteristic bands confirms the wellestablished point of view that cobalt spinel $\mathrm{Co} 3 \mathrm{O} 4$ and copper oxide are the most active in oxidation reactions. The evaluation criterion is the content of peroxide groups, and the standard is a platinum catalyst based on cordierite. Thus, this confirms the hypothesis that the presence of peroxide groups in reactions involving oxygen plays a special role, according to which the peroxide ion is able to migrate along the lattice:

$\mathrm{O}_{2}{ }^{2-}+2 \mathrm{O}^{2-} \rightarrow 2 \mathrm{O}^{2-}+\mathrm{O}_{2}{ }^{2-}$,

and disproportionate with the release of gaseous oxygen:

$$
\mathrm{O}_{2}^{2-}+\mathrm{O}_{2}^{2-} \rightarrow 2 \mathrm{O}^{2-}+\mathrm{O}_{2} \uparrow \text {. }
$$

An important feature is that this process does not require the transfer of an electron from the bulk of the oxide. The absence of peroxide groups in copper oxide and an anomalously high content in nitrous oxide indicates that their appearance is associated with thermal reduction of the oxide, which is confirmed experimentally $\left(\mathrm{CuO}, \mathrm{MoO}_{3}\right.$, $\mathrm{Cr}_{2} \mathrm{O}_{3}, \mathrm{Fe}_{2} \mathrm{O}_{3}, \mathrm{ZnO}, \mathrm{V}_{2} \mathrm{O}_{5}$ ). The obtained result indicates an increased content of excitons in $\mathrm{Cu}_{2} \mathrm{O}$ under normal conditions.

The production of heterogeneous catalysts for deep oxidation of the supported type is associated with the creation of an intermediate layer on the surface of the support, the so-called Wash Coat, usually $\gamma$-alumina containing stabilizing additives $\left(\mathrm{C}\right.$ e $\mathrm{O}_{2}$ ), on the surface of which the catalyst itself is then deposited in the form of a noble metal or oxide phase. In the present work, we used industrial catalysts of domestic production AP$64(0.62 \mathrm{wt} \% \mathrm{Pt}$ on $\gamma$-alumina) and the USA company Walker $(0.2 \mathrm{wt} \% \mathrm{Pt}$ on cordierite 
support). The analysis of catalytic activity was carried out in a stream of nitrogen at a space velocity of $100,000 \mathrm{~h}^{-1}$ and an oxygen concentration of $4.25 \%$, benzene $0.23 \%$. To compare the activity, supported-type complex oxide catalytic systems (carriers: cordierite, silica gel, $\alpha$ - and $\gamma$-aluminum oxides) and bulk catalysts based on cobalt, copper and chromium oxides were tested.

The role of the alumina primer is manifested in the fact that an increase in its concentration on the surface of the support is accompanied by the appearance and growth of the intensity of characteristic bands, which reaches saturation in the region of $3 \mathrm{wt} \%$. The observed result, estimating the minimum size of alumina particles by the minimum size of the transition pores of the support ( $d=50-500 \AA)$, is explained by the manifestation of the size effect ("quantum wells"). The modifying layer is X-ray amorphous, which is probably due to the small particle size. However, the spectrum of the samples allows us to make a conclusion about its structure. The formation of crystalline compounds with a structure close to spinel is accompanied by the appearance of the so-called "spinel" pair of bands, which are attributed to the metal-oxygen stretching vibrations, $630-610$ and $520-500 \mathrm{~cm}^{-1}$. As the concentration of the oxide increases, an increase in the intensity of a series of bands at $530-520 \mathrm{~cm}^{-}{ }^{1}$ and $640-620 \mathrm{~cm}^{-1}$ is recorded in the spectrum, which makes it possible to determine the structure of aluminum oxide particles as a defective spinel ( $\gamma$-oxide).

Cerium and platinum oxides in the catalyst spectrum correspond to mediumintensity bands of vibrational transitions in the range $860-700 \mathrm{~cm}^{-1}$. The results obtained allowed us to conclude that the high catalytic activity is associated with an increased content of highly electronically excited molecular and ionic oxygen associates, primarily, positively charged $\mathrm{O}_{2}{ }^{+}$, which have high diffusion mobility, as well as peroxide groups and exciton states in active phases, carriers, etc. catalytic systems.

\section{Chlorination and methylation of silica surface}

The peculiarities of the modification of the silica surface using initial samples and reagents of various types make it possible to assess the structural and chemical consequences of bulk processes of formation and association of electronically excited states. This follows from the concept of a surface as a macrodefect that concentrates and 
transforms the excitation energy of the hierarchy of bulk defects into the chemical activity of functional groups.

The study of the reaction of silicas of various natures with thionyl chloride vapors showed [29] that the maximum degree of chlorination for all samples is achieved at $200^{\circ} \mathrm{C}$, which is in good agreement with the known data. In this case, the temperature dependence of the concentration of bound chlorine is extreme. The amount of combined chlorine is not proportional to the specific surface area and the concentration of hydroxyl groups.

It is assumed that thionyl chloride is predominantly a nucleophilic agent in the reaction:

$$
\equiv \mathrm{Si}-\mathrm{O}-\mathrm{H}+\mathrm{SOCl}_{2} \equiv \mathrm{Si}-\mathrm{O}-\mathrm{SOCl}+\mathrm{HCl} \uparrow \rightarrow \equiv \mathrm{Si}-\mathrm{Cl}+\mathrm{SO}_{2} \uparrow,
$$

therefore, the substitution of chlorine atoms for hydroxyl groups should tend to unity. The large difference in the degrees of substitution for the used silica preparations and the anomalously low value of $\Theta$ for aerosil indicates a significant difference in the structure (topography) of their surface hydroxyl layer. If we take a cluster of three closely spaced hydroxyls for its elementary fragment, then, taking into account the possibility of the manifestation of the properties of a nucleophilic and electrophilic agent by thionyl chloride, the destruction of the hydroxyl layer is represented by the following scheme: $3(\equiv \mathrm{Si}-\mathrm{OH})+2 \mathrm{SOCl}_{2} \rightarrow(\equiv \mathrm{Si}-\mathrm{O})_{2}=\mathrm{S}=\mathrm{O}+\equiv \mathrm{Si}-\mathrm{O}-\mathrm{SOCl}+2 \mathrm{HCl} \rightarrow$ $\equiv \mathrm{Si}-\mathrm{O}-\mathrm{Si} \equiv+\equiv \mathrm{Si}-\mathrm{Cl}+\mathrm{SO}_{2}$.

The possibility of a frontal nucleophilic attack, which leads to the formation of a $\mathrm{Si}-\mathrm{Cl}$ bond due to intramolecular nucleophilic substitution, is provided by the presence of vacant $3 \mathrm{~d}$ orbitals of silicon atoms. Clustering of hydroxyls provides the degree of their substitution at the level of 0.33 , which practically coincides with the experimentally found for silica gel ShSK. Calculation of the degree of substitution according to the model of the distribution of surface hydroxyl groups of silica gel, proposed by V.F. Kiselev, gives the value $\Theta=0.40$.

The homogenization of the surface due to hydrothermal treatment of silica gel (SG) leads to a significant increase in the degree of substitution, approaching its maximum value. 
Investigation of the surface of silica gel subjected to high-temperature treatment showed that, in this case, there is an increase in the proportion of "paired" clusters that cannot provide the binding of chlorine by silica:

$2(\equiv \mathrm{Si}-\mathrm{OH})+\mathrm{SOCl}_{2} \rightarrow(\equiv \mathrm{Si}-\mathrm{O}) 2=\mathrm{S}=\mathrm{O}++2 \mathrm{HCl} \rightarrow \equiv \mathrm{Si}-\mathrm{O}-\mathrm{Si} \equiv+\mathrm{SO}_{2}$.

The maximum degree of surface chlorination with carbon tetrachloride is achieved at $673 \mathrm{~K}$, the temperature range is limited by the decomposition temperature of $\mathrm{CCl}_{4}(773 \mathrm{~K})$ according to the scheme:

$2(\equiv \mathrm{Si}-\mathrm{OH}) \rightarrow \equiv \mathrm{Si}_{2}=\mathrm{O}+\mathrm{CCl}_{4}+\mathrm{H}_{2} \mathrm{O} \uparrow \rightarrow \equiv \mathrm{Si}-\mathrm{OCCl}_{3}+\equiv \mathrm{Si}-\mathrm{Cl} \rightarrow$

$2(\equiv \mathrm{Si}-\mathrm{Cl})+\mathrm{COCl}_{2} \uparrow$

$\equiv \mathrm{Si}-\mathrm{OH}+\equiv \mathrm{Si}-\mathrm{OCCl}_{3}+\equiv \mathrm{Si}-\mathrm{Cl} \rightarrow \equiv \mathrm{Si}_{2}=\mathrm{O}+\equiv \mathrm{Si}-\mathrm{Cl}+\mathrm{HCl}+\mathrm{COCl}_{2} \uparrow$,

the completeness of chlorination depends on the degree of preliminary dehydration of the surface $\left(\mathrm{K}_{\mathrm{d}}\right)$, which is maximum for A-175 and ShSK.

During surface methylation [31], the presence of one chlorine atom in the trimethylchlorosilane molecule determines the unambiguity of the condensation reaction on the hydroxylated surface according to the scheme:

$$
\mathrm{m}(\equiv \mathrm{Si}-\mathrm{OH})+\mathrm{m} \mathrm{ClSi}\left(\mathrm{CH}_{3}\right)_{3} \rightarrow(\equiv \mathrm{Si}-\mathrm{O}-\mathrm{Si})_{\mathrm{m}}(\mathrm{CH} 3)_{3 \mathrm{~m}}+\mathrm{m} \mathrm{HCl}
$$

The maximum surface coverage achieved for all samples at $200^{\circ} \mathrm{C}$ is minimal for Aerosil and ShSK silica gel. It is almost complete for hydrothermal silica gel. Taking into account the "umbrella" effect of trimethylsilyl groups covering hydroxyls from the electrophilic attack of TMS molecules and clustering of the functional layer, the amount of surface coverage with TMS groups can be represented by the expression:

$$
\Theta_{\text {calc. }}=\alpha_{\mathrm{OH}} \cdot \beta_{\mathrm{OH}} / \alpha_{\mathrm{TMC}} \cdot \beta_{\mathrm{TMC}},
$$

where $\alpha_{\mathrm{OH}}$ is the concentration of hydroxyl groups per $100 \AA^{2} ; \beta \mathrm{OH}$ - effective molecular site of hydroxyl groups; $\alpha_{\text {tms }}$ is the average concentration of TMS groups $\left(2.4 / 100 \AA^{2}\right) ; \beta_{\text {tms }}$ is the molecular site of TMS groups $\left(42 \AA^{2}\right)$. The results confirm the correctness of the chosen model.

The molecular layering method opens up the possibility of eliminating the clustering of the hydroxyl layer by modifying the surface with monomolecular aluminum and titanium oxide layers in the course of polymer-analogous transformations. The 
interaction of the thus obtained silica samples with TMHS is described by the following scheme:

$$
\begin{aligned}
& (\equiv \mathrm{Si}-\mathrm{O}-)_{3} \mathrm{TiOH}+\mathrm{ClSi}\left(\mathrm{CH}_{3}\right)_{3} \rightarrow(\equiv \mathrm{Si}-\mathrm{O}-)_{3} \mathrm{TiO}-\mathrm{Si}\left(\mathrm{CH}_{3}\right)_{3}+\mathrm{HCl} \\
& (\equiv \mathrm{Si}-\mathrm{O}-)_{2} \mathrm{AlOH}+\mathrm{ClSi}\left(\mathrm{CH}_{3}\right)_{3} \rightarrow(\equiv \mathrm{Si}-\mathrm{O}-)_{2} \mathrm{AlO}-\mathrm{Si}\left(\mathrm{CH}_{3}\right)_{3}+\mathrm{HCl} .
\end{aligned}
$$

In all cases, the result is a dense trimethylsilyl layer.

Comparing the results obtained with the thermal emission activity and the chemical structure of excited states in the samples under study, we can conclude that the processes of association of the latter significantly change the topography of the surface functional layer by clustering it.

\section{Catalytic synthesis of carbon}

Studies of the synthesis of carbon layers by the molecular layering method [30] and the mechanism of interaction of carbon tetrachloride with a hydroxylated silica surface served as the basis for the development of a method for the synthesis of carbon layers from a mixture of carbon tetrachloride and hydrocarbons [31].

The study of the formation of carbon on the surface of silica was carried out in a temperature range of $200-450^{\circ} \mathrm{C}$ in an inert environment, under conditions when there is practically no molecularly bound water on the surface of silica gel, and carbon tetrachloride and hydrocarbons themselves do not undergo thermal decomposition.

To assess the role of structural excitations in the synthesis process, active alumina was used.

Since $\mathrm{CCl}_{4}$ is one of the most effective telogenes, and peroxides and oxygen are typical traditional initiators of telomerization, the process of radical telomerization seems to be the basis of the synthesis mechanism [32].

Studies have shown that the reaction of a mixture of $\mathrm{CCl}_{4}-\mathrm{CH}_{4}$ with silica gel begins at about $200^{\circ} \mathrm{C}$, which corresponds to the temperatures of the initiating action of oxygen and peroxides $\left(175-240^{\circ} \mathrm{C}\right)$. Analysis of the synthesis products and the kinetics of their formation showed a high completeness of the heterogeneous polycondensation process according to the following scheme:

$$
\mathrm{CCl}_{4}+\mathrm{CH}_{4}=2 \mathrm{C}+4 \mathrm{HCl} \text {. }
$$


The results of the analysis of the composition of the synthesized carbon layer, as well as the electronic and X-ray photoelectron spectra of the samples, made it possible to evaluate the nature of its bond with the surface and the type of structure.

Since carbon tetrachloride is one of the most effective telogenes, and a methanechemically stable molecule, the most probable scheme of the process is the coordinationradical pathway of polycondensation (radical telomerization), in which excited surface bridging bonds act as a radical initiator:

$$
\begin{aligned}
& \equiv \mathrm{Si}-\mathrm{O}-\mathrm{CCl}_{3} \rightarrow \equiv \mathrm{Si}-\mathrm{O}-\mathrm{CCl}_{2} \cdot+\mathrm{Cl} \\
& \quad \mathrm{Cl} \cdot+\mathrm{CH}_{4} \rightarrow \mathrm{CH}_{3} \cdot+\mathrm{HCl} \\
& \equiv \mathrm{Si}-\mathrm{O}-\mathrm{CCl}_{2} \cdot+\mathrm{CH}_{3} \cdot \rightarrow \equiv \mathrm{Si}-\mathrm{O}-\mathrm{CCl}_{2} \mathrm{CH}_{3} \rightarrow \equiv \mathrm{Si}-\mathrm{O}-\mathrm{C} \equiv \mathrm{CH}+2 \mathrm{HCl} \\
& \quad \equiv \mathrm{Si}-\mathrm{O}-\mathrm{C} \equiv \mathrm{CH}+\mathrm{CCl}_{4} \rightarrow \equiv \mathrm{Si}-\mathrm{O}-\mathrm{C} \equiv \mathrm{C}-\mathrm{CCl}_{3}+\mathrm{HCl} .
\end{aligned}
$$

The activation energy of $\sim 50 \mathrm{~kJ} / \mathrm{mol}$ corresponds to the radical mechanism of limiting stages.

The use of $\gamma-\mathrm{Al}_{2} \mathrm{O}_{3}$ showed that, in this case, the reaction zone moves into the gas phase with the formation of various (colored) telomerization products, which condense in the cold zone of the reactor. The rate of formation of carbon layers is negligible.

A characteristic feature of the spectrum of the synthesized samples is an increased intensity of singlet oxygen lines relative to the initial silica gel. The effect is due to the fullerene-like structure of the synthesized layers.

\section{Results}

1. The concept of thermal generation of electronically excited states in the structure of oxides, based on the concept of the structural and chemical conditions for the accumulation of vibrational energy of structural deformations, vibronic interactions, and exoenergetic stages of the charge transfer process, has been experimentally confirmed. The concept is confirmed by the registration of electronically excited states of the structural bonds of Me-O, their homolytic decomposition and intramolecular rearrangements, which ensure the stabilization of the products of redox reactions due to the association of chemical structure defects. 
2. It was shown for the first time that the main components of gas samples thermally desorbed by oxides are electronically excited forms of molecular oxygen. The process of their generation is volumetric and is accompanied by the accumulation of metal clusters in the structure of oxides.

3. It was found that in the process of oxygen thermionic emission there is a dependence of the specific activity of the samples on their mass, which is of a nonlinear nature. The kinetic and thermodynamic models of the process confirm that the nature of the effect is due to the presence of electronically excited oxygen molecules.

4. For the first time, spectral methods have established the existence of electronically excited states in the structure of oxides under dark conditions at standard and elevated temperatures. The chemical structure of the components of electronic excitation in the oxygen and cationic sublattices is characterized by isolated associates of the molecular type. They correspond to compounds of oxidized oxygen anions, which include a series of triplet and singlet molecules, ozone, per- and superoxide groups, as well as excited quasi-molecular metal-metal bonds. The concentration of excited states reaches and exceeds the threshold level of $10^{17}-10^{18} \mathrm{~cm}^{-3}$.

5. For the first time, the fact of the occurrence of cooperative interaction of electronically excited states under standard conditions and at elevated temperatures has been established. It was found that the intensity of IR luminescence accompanying the process is due to the size effect, which corresponds to the antibate dependence of the intensity on the characteristic size of structural elements.

6. It is shown that the high catalytic activity of oxide systems in deep oxidation reactions of hydrocarbons is associated with anomalously high thermionic emission of electronically excited states of molecular oxygen. It was found that in the structure it corresponds to a high concentration of electronically excited states of the exciton type, an increased content of metal clusters, a high concentration of diffusionally mobile peroxide groups, a high concentration of positively charged oxygen radical ions, and an increased content of highly excited forms of molecular oxygen and ozone.

7. A new method for obtaining condensed carbon structures on the surface of oxides has been developed. It is shown that the high concentration and mobility of electronically excited states in the structure of active alumina exclude the possibility of 
polycondensation polymerization on the surface. The large-scale association and stabilization of excitations in the case of $\mathrm{SiO}_{2}$ creates conditions for the conversion of bulk radical telomerization processes into a heterogeneous polymerization process. 8. It is shown that the processes of association of exciton states determine the topography of the layer of functional groups on the surface of silicon oxides, which manifests itself in the completeness of the reactions of chlorination and methylation of the surface.

\section{Conclusions}

The effect and regularities of large-scale dark generation of excitons in oxide systems and the possibility of its regulation open up prospects for the production of new highly efficient materials. First of all, we can talk, along with catalysts for deep and partial oxidation in the processing of hydrocarbon raw materials and environmental protection, about catalytic systems for the production of alternative ecological fuels such as hydrogen and ammonia. In this case, compounds of the hydride and nitride series can be used as the basis for the catalytic action.

Taking into account the discovered anomalous quantum features of the behavior of exciton states at concentrations above the threshold and elevated temperatures, which, as has been shown, are quite attainable by traditional chemical methods, one can count on the synthesis of superconducting materials that are stable at normal and elevated temperatures.

Bulk generation of Coulomb excitons is accompanied by processes of their diffusionactivated transport to the surface of the catalytically active phase, where they dissociate into electrons and holes. Thus, the process of charge separation is implemented, which underlies the operation of modern power sources. The latter have a number of significant disadvantages associated with high resistance of electrolytes and polarization of electrodes. The use of a gaseous medium (air) as an electrolyte as a carrier of positively charged holes, which are emitted by a catalytically active anode, reduces the internal resistance of such an element to practically zero. A catalytically active cathode with electronic conductivity receives electrons through an external (working) circuit. Thus, a schematic of operation of a fuel cell is realized, in which a variety of external energy sources can be used as fuel, including organic, hydrogen, solar, and all types of radiation 
activation. In the latter case, catalytic deactivation of the used radionucleides is possible as one of the ways of processing nuclear waste. An experimental estimate of the output parameters of such a scheme shows the possibility of achieving current values at the level of 1-10 A $/ \mathrm{cm}^{2}$ and EMF at the level of 5-100 V, which are in good agreement with the data on the concentration of excitons and the rate of their decomposition on the anode surface.

Such a scheme may well be implemented in the version of a renewable storage of electrical energy, which can have a huge capacity, limited only by the capacity of the superconducting layer of the exciton condensate.

\section{References}

1. Korolkov D.V., Electronic structure and properties of compounds of non-transition elements.- SPb .: Chemistry, 1992. -312 p.

2. Krylov OV Kiselev V.F., Adsorption and catalysis on transition metals and their oxides. - M .: Chemistry, 1981 . -288 p. Bersuker I.B.

3. A.S. USSR No. 400168. Method for producing phosgene. Kopylov V.B., Koltsov S.I., Smirnov V.M., Aleskovsky V.B., 1972

4. RF patent No. 2002498. Kopylov VB, Pak VN, Turbin AS. Catalyst for cleaning gases from carbon monoxide and organic matter. 1993

5. Koltsov SI, Smirnov EP, Kopylov VB, Aleskovsky VB, Molecular carbon layering on silica adsorbents of various porous structures. Sat. Adsorbents, their properties and application. 1978.-L .: Nauka.- S. 56-60.

6. Bersuker I.B., Electronic structure and properties of coordination compounds. Leningrad: Chemistry, 1986.- 287 p.

7. Bersuker I.B., Jahn-Teller effect and vibronic interactions in modern chemistry. -M .: Nauka, 1987.-344p.

8. Volkenstein F.F., Physics and chemistry of semiconductor surfaces.- Moscow: Nauka. 1973.-399 p.

9. Plakida NM, High-temperature superconductors. M. International Education Program. 1996 
10. Kopylov VB, Pushkar IV, Gavronskaya Yu.Yu., Local structural excitations and critical temperature of deposited superconducting oxides. // Journal of General Chemistry, 2004. Vol. 74, No. 5. P. 864-865.

11. Belousov M.A., Davydov V.Yu., Raman scattering of light as a method for studying the oxygen sublattice of high-temperature superconductors. Sat. High temperature superconductivity. Part 2.-L.: Leningrad State University, 1989.- P.6-29.

12. Vinogradov B.A., Kopylov V.B., Boyarkin K.E., Photoacoustic method for studying the dynamics of fracture of materials. // Sat. Introduction of highly efficient technological processes using lasers in industry during the implementation of the "Intensification -90" program. L .: LDNTP. 1986.- S. 76-80.

13. Kroger F.A., The chemistry of imperfect crystals. 2nd ed. - Amsterdam: NorthHolland Publ. Co, 1974 .- 242 p.

14. Anderson J.S., Problems of nonstoichiometry.- Amsterdam: North-Holland Publ. Co, 1969, -212 p.

15. Aria S.M., Thermochemistry of oxides and chalcogenides of variable composition and their magnetic properties // In collection: Compounds of variable composition.Leningrad: Chemistry, 1969.-169 p.

16. Belova I.D., Zavyalov S.A., Roginskaya Yu.E., Emission of singlet oxygen molecules from the surface of Soz.xO4 films. // Journal of Physical Chemistry. 1986. T. 60, No. 9.S. $2338-2340$.

17. Kiryanov N.V., Borets V.N., Morozov Yu.G., Grigoryan E.A., Features of the kinetics of oxygen evolution into vacuum from ceramics of composition YBa2Cu3O7-x. // Journal. Phys. Chem. 1996 T. 70, No. 2.- P. 311-315.

18. Kopylov V.B., Loseva N.I., Pak V.N., Desorption and thermal emission of oxygen from the glass surface. // Journal of Applied Chemistry, 1996. Vol. 69, No. 6. S. 926-930. 19. Kopylov V.B., Loseva N.I., Pak V.N., Features of thermal emission of oxygen by aluminosilicate ceramics. // Journal of Applied Chemistry., 1996. Vol. 69, No. 11. S. 1852-1856.

20. Kopylov V.B., Loseva N.I., Gas chromatographic separation of components of a mixture desorbed from the surface of glasses. // Journal. Phys. Chemistry, 1998, Vol. 72, No. 12. S. 2226-2229. 
21. Kopylov V.B., Thermal effects of sorption and desorption of molecular oxygen on an oxidized carbon surface. // Journal. Phys. Chemistry, 2001. T.75, 6. - S.1045-1048.

22. Glensdorf P., Prigogine I., Thermodynamic theory of structure, stability and fluctuations.- Moscow: Mir, 1973. -285 p.

23. Sokolov I.M., Dimensions and other geometric critical exponents in percolation theory. // Usp. Phys. Sciences, 1986, Vol. 150. - S. 221.

24. Berreman D.W., Infrared absorption at longitudinal optic frequency in cubic crystals films. // Phys. Rev. 1963. V.130. No. 6.- P.2193-2196.

25. Razumovsky S. D., Oxygen. Elementary forms and properties. -M .: Chemistry, 1979.- S. 186-225.

26. Knox R., Theory of excitons.- Moscow: Mir, 1966.- 217 p.

27. Kopylov V.B., Aleksandrov K.A., Sergeev E.V., Thermoevolution of the chemical structure and properties of silicon oxides. // Journal. Common Chemistry, 2008. T. 78, No. 5. S. 726 - 733.

28. Kopylov V.B., Yakovlev I.A., Influence of the nature of aluminum oxide on the oxygen state of its surface. // Journal of General Chemistry, 2001. Vol. 71, issue 4. S. 684-685.

29. Kopylov V.B., Tsvetkov V.K., Pak V.N., Influence of the nature of silica on the chlorination of their surface. // Journal. Applied Chemistry, 1983, T. 56, No. 5.P. 1234 1238.

30. Koltsov S.I., Smirnov E.P., Kopylov V.B., Aleskovsky V.B., Molecular carbon layering on silica adsorbents of various porous structures. // Sat. Adsorbents, their properties and applications. 1978. Leningrad. The science. S. 56-60.

31. A.S. No. 740730 USSR. Refractory board processing method. / Smirnov E.P., Kopylov V.B., Zhukov S.G., Koltsov S.I., Nikolaev A.R., Kortel A.A., Aleskovsky V.B. 1980. BI number 22.

32. Freindlina R.Kh., Velichko F.K., Zlotsky S.S., Rakhmankulov D.L., Terentyev A.B. Radical telomerization. - M .: Chemistry, 1988. - 288 